\title{
Weak Poincaré Inequalities for Convergence Rate of Degenerate Diffusion Processes *
}

\author{
Martin Grothaus ${ }^{b)}$ and Feng-Yu Wang ${ }^{a), c)}$ \\ a) Center for Applied Mathematics, Tianjin University, Tianjin 300072, China \\ b) Mathematics Department, Kaiserslautern University, 67653 Kaiserslautern, Germany \\ c) Department of Mathematics, Swansea University, Singleton Park, SA2 8PP, UK \\ grothaus@mathematik.uni-kl.de; wangfy@bnu.edu.cn, F.-Y.Wang@swansea.ac.uk
}

10 December 2018

\begin{abstract}
For a contraction $C_{0}$-semigroup on a separable Hilbert space, the decay rate is estimated by using the weak Poincaré inequalities for the symmetric and anti-symmetric part of the generator. As applications, non-exponential convergence rate is characterized for a class of degenerate diffusion processes, so that the study of hypocoercivity is extended. Concrete examples are presented.
\end{abstract}

AMS subject Classification: 60J10, 47D07.

Keywords: Degenerate diffusion semigroup, hypocercivity, weak Poincaré inequality, convergence rate.

\section{Introduction}

Let $(E, \mathscr{F}, \mu)$ be a probability space and let $(\mathscr{E}, \mathscr{D}(\mathscr{E}))$ be the quadratic form associated with a Markov semigroup $P_{t}$ on $L^{2}(\mu)$. The weak Poincaré inequality

$$
\operatorname{Var}_{\mu}(f):=\mu\left(f^{2}\right)-\mu(f)^{2} \leq \alpha(r) \mathscr{E}(f, f)+\alpha(r)\|f\|_{\text {osc }}^{2}, \quad r>0, f \in \mathscr{D}(\mathscr{E})
$$

with rate function $\alpha:(0, \infty) \rightarrow(0, \infty)$ was introduced in [20] to describe the following convergence rate of $P_{t}$ to $\mu$ :

$$
\xi(t):=\sup _{\|f\|_{\text {osc }} \leq 1} \operatorname{Var}_{\mu}\left(P_{t} f\right), \quad t>0 .
$$

${ }^{*}$ Supported in part by NNSFC $(11431014,11626245,11626250)$. 
Explicit correspondence between $\alpha$ and $\xi$ has been presented in [20]. In particular, the weak Poincaré inequality (1.1) is always available for elliptic diffusion processes. However, it does not hold when the Dirichlet form is reducible. A typical example is the stochastic Hamiltonian system on $\mathbb{R}^{d} \times \mathbb{R}^{d}$ :

$$
\left\{\begin{array}{l}
\mathrm{d} X_{t}=Y_{t} \mathrm{~d} t \\
\mathrm{~d} Y_{t}=\sqrt{2} \mathrm{~d} B_{t}-\left(\nabla^{(1)} V\left(X_{t}\right)+Y_{t}\right) \mathrm{d} t,
\end{array}\right.
$$

where $B_{t}$ is the Brownian motion on $\mathbb{R}^{d}, \nabla^{(1)}$ is the gradient operator in the first component $x \in \mathbb{R}^{d}$, and $V \in C^{2}\left(\mathbb{R}^{d}\right)$ satisfies

$$
\left\|\nabla^{2} V\right\| \leq M(1+|\nabla V|)
$$

for some constant $M>0$ and $Z(V):=\int_{\mathbb{R}^{d}} \mathrm{e}^{-V(x)} \mathrm{d} x<\infty$. In this case the invariant probability measure of the diffusion process is $\mu=\mu_{1} \times \mu_{2}$, where $\mu_{1}(\mathrm{~d} x)=Z(V)^{-1} \mathrm{e}^{-V(x)} \mathrm{d} x$ and $\mu_{2}$ is the standard Gaussian measure on $\mathbb{R}^{d}$. Let $\nabla^{(2)}$ be the gradient operator in the second component $y \in \mathbb{R}^{d}$. Then the associated energy form satisfies $\mathscr{E}(f, f)=\mu\left(\left|\nabla^{(2)} f\right|^{2}\right)$, and is thus reducible.

On the other hand, according to C. Villani [24], if the Poincaré inequality

$$
\operatorname{Var}_{\mu_{1}}(f):=\mu_{1}\left(f^{2}\right)-\mu_{1}(f)^{2} \leq c_{1} \mu_{1}\left(|\nabla f|^{2}\right), \quad f \in C_{b}^{1}\left(\mathbb{R}^{d}\right)
$$

holds for some constant $c_{1}>0$, then the Markov semigroup $P_{t}$ associated with (1.2) converges exponentially to $\mu$ in the sense that

$$
\mu\left(\left|P_{t} f-\mu(f)\right|^{2}+\left|\nabla P_{t} f\right|^{2}\right) \leq c_{2} \mathrm{e}^{-\lambda t} \mu\left(|f-\mu(f)|^{2}+|\nabla f|^{2}\right), \quad t \geq 0, f \in C_{b}^{1}\left(\mathbb{R}^{d}\right)
$$

holds for some constants $c_{2}, \lambda>0$, where and in the following, $\mu(f):=\int f \mathrm{~d} \mu$ for $f \in L^{1}(\mu)$. If the gradient estimate $\left|\nabla P_{t} f\right|^{2} \leq K(t) P_{t} f^{2}$ holds for some function $K:(0, \infty) \rightarrow(0, \infty)$, see $[15,26]$ for concrete estimates, we obtain the $L^{2}$-exponential convergence

$$
\operatorname{Var}_{\mu}\left(P_{t} f\right) \leq c \mathrm{e}^{-\lambda t} \operatorname{Var}_{\mu}(f), \quad t \geq 0, f \in L^{2}(\mu)
$$

for some constants $c, \lambda>0$, which has been derived in [12] using the idea of [8]. See e.g. $[1,8,10,11,12,15,25,26]$ and references within for further results on exponential convergence and regularity estimates of $P_{t}$.

Recently, $\mathrm{Hu}$ and Wang [16] prove the sub-exponential convergence by using the weak Poincaré inequality

$$
\operatorname{Var}_{\mu_{1}}(f) \leq \alpha(r) \mu_{1}\left(|\nabla f|^{2}\right)+r\|f\|_{\text {osc }}^{2}, \quad f \in C_{b}^{1}\left(\mathbb{R}^{d}\right)
$$

for some decreasing function $\alpha:(0, \infty) \rightarrow(0, \infty)$, where $\|f\|_{\text {osc }}:=\operatorname{ess}_{\mu} \sup f-\operatorname{ess}_{\mu} \inf f$. According to [16, Theorem 3.6], (1.6) implies

$$
\mu\left(\left|P_{t} f-\mu(f)\right|^{2}+\left|\nabla P_{t} f\right|^{2}\right) \leq c_{1} \xi(t)\left(\|f\|_{\infty}^{2}+\mu\left(|\nabla f|^{2}\right)\right), \quad t \geq 0, f \in C_{b}^{1}\left(\mathbb{R}^{d}\right)
$$


for some constant $c_{2}>0$ and

$$
\xi(t):=\inf \{s>0: t \geq-\alpha(s) \log s\}, \quad t \geq 0 .
$$

Again, if the gradient estimate $\left|\nabla P_{t} f\right|^{2} \leq K(t) P_{t} f^{2}$ holds then this implies

$$
\operatorname{Var}_{\mu}\left(P_{t} f\right) \leq c_{1} \xi(t)\|f\|_{\text {osc }}^{2}, \quad t \geq 0, f \in L^{\infty}(\mu)
$$

for some constant $c_{1}>0$. In particular, if $\alpha$ is bounded so that (1.6) reduces to (1.4) with $c_{1}=\|\alpha\|_{\infty}$, we obtain the exponential convergence as in the previous case.

In this paper we aim to introduce weak Poincaré inequalities to estimate the convergence rate for more general degenerate diffusion semigroups where $\mu_{2}$ is not necessarily a Gaussian measure. Consider the following degenerate SDE for $\left(X_{t}, Y_{t}\right)$ on $\mathbb{R}^{d_{1}+d_{2}}=\mathbb{R}^{d_{1}} \times \mathbb{R}^{d_{2}}$, where $d_{1}, d_{2} \geq 1$ may be different:

$$
\left\{\begin{array}{l}
\mathrm{d} X_{t}=Q\left(\nabla^{(2)} V_{2}\right)\left(Y_{t}\right) \mathrm{d} t \\
\mathrm{~d} Y_{t}=\sqrt{2} \mathrm{~d} B_{t}-\left(Q^{*}\left(\nabla^{(1)} V_{1}\right)\left(X_{t}\right)+\left(\nabla^{(2)} V_{2}\right)\left(Y_{t}\right)\right) \mathrm{d} t,
\end{array}\right.
$$

where $Q$ is a $d_{1} \times d_{2}$-matrix, $V_{i} \in C^{2}\left(\mathbb{R}^{d_{i}}\right)$ such that $Z\left(V_{i}\right)<\infty, i=1,2$, and $\nabla^{(1)}, \nabla^{(2)}$ are the gradient operators in components $x \in \mathbb{R}^{d_{1}}$ and $y \in \mathbb{R}^{d_{2}}$ respectively. It is easy to see that the generator of solutions to (1.9) is dissipative in $L^{2}(\mu)$, where $\mu:=\mu_{1} \times \mu_{2}$ for probability measures $\mu_{i}(\mathrm{~d} x):=Z\left(V_{i}\right)^{-1} \mathrm{e}^{-V_{i}(x)} \mathrm{d} x$ on $\mathbb{R}^{d_{i}}, i=1$, 2; see the beginning of Section 3 for details.

Since the coefficients of the SDE (1.9) are locally Lipschitz continuous, for any initial point $z=(x, y) \in \mathbb{R}^{d_{1}+d_{2}}$, the SDE has a unique solution $\left(X_{t}^{z}, Y_{t}^{z}\right)$ up to life time $\zeta^{z}$. Let $P_{t}$ be the associated (sub-) Markov semigroup, i.e.

$$
P_{t} f(z)=\mathbb{E}\left[f\left(X_{t}^{z}, Y_{t}^{z}\right) 1_{\left\{t<\zeta^{z}\right\}}\right], \quad f \in \mathscr{B}_{b}\left(\mathbb{R}^{d_{1}+d_{2}}\right), z \in \mathbb{R}^{d_{1}+d_{2}}, t \geq 0 .
$$

To ensure the non-explosion of the solution and the convergence of the $L^{2}$-Markov semigroup $P_{t}$ to $\mu$, we make the following assumption.

(H) $Q Q^{*}$ is invertible, there exists a constant $M>0$ such that

$$
\left|\left(\nabla^{(i)}\right)^{2} V_{i}\right| \leq M\left(1+\left|\nabla^{(i)} V_{i}\right|^{\tau_{i}}\right), \quad i=1,2,
$$

for $\tau_{1}=1$ and some $1 \leq \tau_{2}<2$. Moreover, $\mu_{2}\left(\left|\nabla^{(2)} V_{2}\right|^{4}\right)<\infty$ and $V_{2}(y)=\Phi\left(|\sigma y-b|^{2}\right)$ for some invertible $d_{2} \times d_{2}$-matrix $\sigma, b \in \mathbb{R}^{d_{2}}$ and increasing function $\Phi \in C^{3}([0, \infty))$ such that

$$
\sup _{r \geq 0}\left|\Phi^{\prime}(r)+2 r \Phi^{\prime \prime}(r)-\frac{2 r \Phi^{\prime \prime \prime}(r)+\left(d_{2}+2\right) \Phi^{\prime \prime}(r)}{\Phi^{\prime}(r)}\right|<\infty
$$

According to [20, Theorem 3.1], there exist two decreasing functions $\alpha_{1}, \alpha_{2}:(0, \infty) \rightarrow$ $[1, \infty)$ such that the weak Poincaré inequality

$$
\operatorname{Var}_{\mu_{i}}(f) \leq \alpha_{i}(r) \mu_{i}\left(\left|\nabla^{(i)} f\right|^{2}\right)+r\|f\|_{\text {osc }}^{2}, \quad f \in C_{b}^{1}\left(\mathbb{R}^{d_{i}}\right), r>0,
$$

holds for $i=1,2$. We have the following result on the convergence rate of $P_{t}$ to $\mu$. 
Theorem 1.1. Let $V_{1}$ and $V_{2}$ satisfy $(H)$. Then the solution to (1.9) is non-explosive and $\mu$ is an invariant probability measure of the associated Markov semigroup $P_{t}$. Moreover, there exist constants $c_{1}, c_{2}>0$ such that (1.8) holds for

$$
\xi(t):=c_{1} \inf \left\{r>0: c_{2} t \geq \alpha_{1}(r)^{2} \alpha_{2}\left(\frac{r}{\alpha_{1}(r)^{2}}\right) \log \frac{1}{r}\right\},
$$

which goes to 0 as $t \rightarrow \infty$.

Remark 1.2. (1) When $V_{2}(y)=\frac{1}{2}|y|^{2}$ the measure $\mu_{2}$ reduces to the standard Gaussian measure as in [16]. In this case, we may repeat the argument in the proof of [16, Theorem 3.6] to prove (1.7) for

$$
\xi(t)=\inf \left\{r>0: c_{2} t \geq \alpha_{1}(r) \log \frac{1}{r}\right\}, \quad t>0,
$$

and thus extend the main result in [16] to the case that $d_{1} \neq d_{2}$. Since in this case we have $\alpha_{2} \equiv 1$, the convergence rate in Theorem 1.1 becomes

$$
\xi(t)=\inf \left\{r>0: c_{2} t \geq \alpha_{1}(r)^{2} \log \frac{1}{r}\right\}, \quad t>0,
$$

which is in general worse than that in (1.14). However, the argument in [16] heavily depends on the specific $V_{2}(y)=\frac{1}{2}|y|^{2}$ (or by linear change of variables $V_{2}(y)=|\sigma y-b|^{2}$ for some invertible $d_{2} \times d_{2}$-matrix $\sigma$ and $b \in \mathbb{R}^{d_{2}}$ ), and is hard to extend to a general setting as in $(H)$. Nevertheless, we would hope to improve the convergence rate in Theorem 1.1 such that (1.14) is covered for bounded $\alpha_{2}$.

(2) Theorem 1.1 also applies to the following SDE for $\left(X_{t}, \bar{Y}_{t}\right)$ on $\mathbb{R}^{d_{1}+d_{2}}$ for some invertible $d_{2} \times d_{2}$-matrix $\sigma$ and invertible $d_{1} \times d_{1}$-matrix $\bar{Q} \bar{Q}^{*}$ :

$$
\left\{\begin{array}{l}
\mathrm{d} X_{t}=\bar{Q}\left(\nabla^{(2)} V_{2}\right)\left(\bar{Y}_{t}\right) \mathrm{d} t \\
\mathrm{~d} \bar{Y}_{t}=\sqrt{2} \sigma \mathrm{d} B_{t}-\left(\bar{Q}^{*}\left(\nabla^{(1)} V_{1}\right)\left(X_{t}\right)+\sigma \sigma^{*}\left(\nabla^{(2)} V_{2}\right)\left(\bar{Y}_{t}\right)\right) \mathrm{d} t .
\end{array}\right.
$$

Indeed, let $\left(X_{t}, Y_{t}\right)$ solve (1.9) and let $\bar{Y}_{t}=\sigma Y_{t}, \bar{V}_{2}(y)=V_{2}\left(\sigma^{-1} y\right)$. We have

$$
\left(\nabla^{(2)} \bar{V}_{2}\right)(y)=\left(\sigma^{-1}\right)^{*}\left(\nabla^{(2)} V_{2}\right)\left(\sigma^{-1} y\right), \quad y \in \mathbb{R}^{d_{2}},
$$

so that

$$
\mathrm{d} X_{t}=Q\left(\nabla^{(2)} V_{2}\right)\left(Y_{t}\right) \mathrm{d} t=Q \sigma^{*}\left(\nabla^{(2)} \bar{V}_{2}\right)\left(\bar{Y}_{t}\right)
$$

and

$$
\mathrm{d} \bar{Y}_{t}=\sqrt{2} \sigma \mathrm{d} B_{t}-\left(\sigma Q^{*}\left(\nabla^{(1)} V_{1}\right)\left(X_{t}\right)+\sigma \sigma^{*}\left(\nabla^{(2)} \bar{V}_{2}\right)\left(\bar{Y}_{t}\right)\right) \mathrm{d} t .
$$

Letting $\bar{Q}=Q \sigma^{*}$, we see that the $\operatorname{SDE}(1.9)$ is equivalent to (1.15).

To illustrate Theorem 1.1, we consider the following example with some concrete convergence rates of $P_{t}$. 
Example 1.3. We write $f \sim g$ for real functions $f$ and $g$ on $\mathbb{R}^{d}$ if $f-g \in C_{b}^{2}\left(\mathbb{R}^{d}\right)$.

(A) Let $V_{1}(x) \sim k\left(1+|x|^{2}\right)^{\frac{\delta}{2}}$ for some constants $k, \delta>0$.

$\left(A_{1}\right)$ When $V_{2}(y)=\kappa\left(1+|y|^{2}\right)^{\frac{\varepsilon}{2}}$ for some constants $\kappa, \varepsilon>0$, (1.8) holds with

$$
\xi(t)=c_{1} \exp \left(-c_{2} t^{\frac{\varepsilon \delta}{\varepsilon \delta+8 \varepsilon(1-\delta)^{+}+4 \delta(1-\varepsilon)^{+}}}\right), \quad t \geq 0,
$$

for some constants $c_{1}, c_{2}>0$. If, in particular, $\delta, \varepsilon \geq 1$ then $P_{t}$ converges to $\mu$ exponentially fast.

$\left(A_{2}\right)$ When $V_{2}(y)=\frac{d+p}{2} \log \left(1+|y|^{2}\right)$ for some constant $p>0,(1.8)$ holds with

$$
\xi(t)=c(1+t)^{-\frac{1}{\theta(p)}}(\log (\mathrm{e}+t))^{\frac{8(\theta(p)+1)(1-\delta)^{+}+\delta}{\theta(p) \delta}}
$$

for some constant $c>0$ and

$$
\theta(p):=\frac{d+p+2}{p} \wedge \frac{4 p+4+2 d}{\left(p^{2}-4-2 d-2 p\right)^{+}} .
$$

$\left(A_{3}\right)$ When $V_{2}(y)=\frac{d}{2} \log \left(1+|y|^{2}\right)+p \log \log \left(\mathrm{e}+|y|^{2}\right)$ for some constants $p>1,(1.8)$ holds with

$$
\xi(t)=c_{1}(\log (\mathrm{e}+t))^{1-p} \cdot\left(\log \log \left(\mathrm{e}^{2}+t\right)\right)^{\frac{8(1-\delta)^{+}}{\delta}}
$$

for some constants $c>0$.

(B) Let $V_{1}(x) \sim \frac{d+q}{2} \log \left(1+|x|^{2}\right)$ for some $q>0$.

$\left(B_{1}\right)$ When $V_{2}(y)=k\left(1+|y|^{2}\right)^{\frac{\varepsilon}{2}}$ for some constants $k, \varepsilon>0$, (1.8) holds with

$$
\xi(t)=c(1+t)^{-\frac{1}{2 \theta(q)}}(\log (\mathrm{e}+t))^{\frac{4(1-\varepsilon)^{+}+\varepsilon}{2 \varepsilon \theta(q)}}
$$

for some constant $c>0$.

$\left(B_{2}\right)$ When $V_{2}(y)=\frac{p+d}{2} \log \left(1+|y|^{2}\right)$ for some constant $p>0,(1.8)$ holds with

$$
\xi(t)=c(1+t)^{-\frac{1}{2 \theta(q)+\theta(p)+2 \theta(p) \theta(q)}}(\log (\mathrm{e}+t))^{\frac{1}{2 \theta(q)+\theta(p)+2 \theta(p) \theta(q)}}
$$

for some constant $c>0$.

$\left(B_{3}\right)$ When $V_{2}(y)=\frac{d}{2} \log \left(1+|y|^{2}\right)+p \log \log \left(\mathrm{e}+|y|^{2}\right)$ for some constant $p>1$, (1.8) holds with

$$
\xi(t)=c(\log (\mathrm{e}+t))^{-\frac{p-1}{1+2 \theta(q)}}
$$

for some constant $c>0$. 
(C) Let $V_{1}(x) \sim \frac{d}{2} \log \left(1+|x|^{2}\right)+q \log \log \left(\mathrm{e}+|x|^{2}\right)$ for some $q>0$.

$\left(C_{1}\right)$ When $V_{2}(y)=k\left(1+|y|^{2}\right)^{\frac{\varepsilon}{2}}$ for some constant $k>0$ and $\varepsilon>0$, or $V_{2}(y)=\frac{p+d}{2} \log \left(1+|y|^{2}\right)$ for some constant $p>0$, (1.8) holds with

$$
\xi(t)=c(\log (\mathrm{e}+t))^{-(q-1)}
$$

for some constant $c>0$.

$\left(C_{2}\right)$ When $V_{2}(y)=\frac{d}{2} \log \left(1+|y|^{2}\right)+p \log \log \left(\mathrm{e}+|y|^{2}\right)$ for some constant $p>1$, (1.8) holds with

$$
\xi(t)=c\left(\log \log \left(\mathrm{e}^{2}+t\right)\right)^{-(q-1)}
$$

for some constant $c>0$.

In the next section we present a general result on the weak hypocoercivity for $C_{0^{-}}$ semigroups on Hilbert spaces, see Theorem 2.1, below. In Section 3 this result is used to prove Theorem 1.1 and Example 1.3. Theorem 2.1 is the main result of this article. It applies to a much larger class of degenerate SDEs as given in (1.9). The state space of the Markov process associated to the semigroup can be very general. For example it could be a manifold or an infinite dimensional space. In particular it also applies to degenerate spherical velocity Langevin equations as treated in [12]. Those are prescribed by manifold-valued Stratonovich stochastic differential equations with state space $\mathbb{M}=\mathbb{R}^{d} \times \mathbb{S}$ of the form

$$
\begin{aligned}
\mathrm{d} x_{t} & =\omega_{t} \mathrm{dt} \\
\mathrm{d} \omega_{t} & =-\frac{1}{d-1}\left(I-\omega_{t} \otimes \omega_{t}\right) \nabla V\left(x_{t}\right) \mathrm{dt}+\sigma\left(I-\omega_{t} \otimes \omega_{t}\right) \circ \mathrm{d} B_{t} .
\end{aligned}
$$

Here $d \in \mathbb{N}$ with $d \geq 2$. $B$ is a standard $d$-dimensional Brownian motion, $z \otimes y=z y^{T}$ for $z, y \in \mathbb{R}^{d}$ and $y^{T}$ is the transpose of $y . \mathbb{S}=\mathbb{S}^{d-1}$ denotes the unit sphere with respect to the euclidean norm in $\mathbb{R}^{d}$. Moreover $x$ denotes the space variable in $\mathbb{R}^{d}$ and $\omega$ the velocity component in $\mathbb{S} \subset \mathbb{R}^{d}$ and all vectors in euclidean space are understood as column vectors. For a specified class of potentials $V: \mathbb{R}^{d} \rightarrow \mathbb{R}$ and $\sigma>0$ a finite constant, in [12] all assumptions of Theorem 2.1 are checked for the equations as in (1.16). But due to Theorem 2.1, in comparison with [12] we now can weaken the growth condition on $V$, since we need the space component of the corresponding invariant measure only to fulfill a weak Poincaré inequality. Hence, $V$ may be chosen as any potential $V_{1}$ from Example 1.3. Solutions to SDEs as in (1.16) e.g. also appear in industrial mathematics as so-called fiber lay-down processes, see [12] and the references therein. They are used as surrogate models for the production process of non-wovens. For those models the rate of convergence to equilibrium is very much of interest, because this rate is related to the quality of non-wovens. Hence, cases in which empirical measurements indicate slow growing potentials, by our main result, now may be covered also. 


\section{A general framework}

Let $(\mathbb{H},\langle\cdot, \cdot\rangle,\|\cdot\|)$ be a separable Hilbert space, let $(L, \mathscr{D}(L))$ be a densely defined linear operator generating a $C_{0^{-}}$contraction semigroup $P_{t}=\mathrm{e}^{t L}$. We aim to investigate the decay rate of $P_{t}$ of type

$$
\left\|P_{t} f\right\|^{2} \leq \xi(t)\left(\|f\|^{2}+\Psi(f)\right), \quad t \geq 0, f \in \mathscr{D}(L),
$$

where $\xi$ is a decreasing function with $\xi(\infty):=\lim _{t \rightarrow \infty} \xi(t)=0$, and $\Psi: \mathbb{H} \rightarrow[0, \infty]$ is a functional such that the set $\{f \in \mathbb{H}: \Psi(f)<\infty\}$ is dense in $\mathbb{H}$.

\subsection{Main result}

Following the line of e.g. $[8,12]$, we assume that $L$ decomposes into symmetric and antisymmetric part:

$$
L=S-A \text { on } \mathscr{D}
$$

where $\mathscr{D}$ is a core of $(L, \mathscr{D}(L)), S$ is symmetric and $A$ is antisymmetric. Then both $(S, \mathscr{D})$ and $(A, \mathscr{D})$ are closable in $\mathbb{H}$. Let $(S, \mathscr{D}(S))$ and $(A, \mathscr{D}(A))$ be their closures. These two operators are linked to the orthogonal decomposition $\mathbb{H}=\mathbb{H}_{1} \oplus \mathbb{H}_{2}$ in the following assumptions, where

$$
\pi_{i}: \mathbb{H} \rightarrow \mathbb{H}_{i}, \quad i=1,2,
$$

are the orthogonal projections.

$(H 1) \mathbb{H}_{1} \subset \mathcal{N}(S):=\{f \in \mathscr{D}(S): S f=0\}$; that is, $\mathbb{H}_{1} \subset \mathscr{D}(S)$ (hence, $\pi_{2} \mathscr{D} \subset \mathscr{D}(S)$ due to $\mathscr{D} \subset \mathscr{D}(S))$ and $S \pi_{1}=0$.

$(H 2) \pi_{1} \mathscr{D} \subset \mathscr{D}(A)$ (hence, also $\pi_{2} \mathscr{D} \subset \mathscr{D}(A)$ due to $\left.\mathscr{D} \subset \mathscr{D}(A)\right)$ and $\left.\pi_{1} A \pi_{1}\right|_{\mathscr{D}}=0$.

Since $(A, \mathscr{D}(A))$ is closed, antisymmetric and $\pi_{1} \mathscr{D} \subset \mathscr{D}(A),\left(\pi_{1} A, \mathscr{D}(A)\right)$ is closable. Denote the closure by $\left(\pi_{1} A, \mathscr{D}\left(\pi_{1} A\right)\right)$. By $(H 2), A \pi_{1}$ is well defined on $\mathscr{D}$, and by the antisymmetry of $A$,

$$
\left(A \pi_{1}\right)^{*}=\pi_{1} A^{*}=-\pi_{1} A \text { holds on } \mathscr{D} \text {. }
$$

Then $A \pi_{1}$ with domain $\mathscr{D}\left(A \pi_{1}\right):=\left\{f \in \mathbb{H}: \pi_{1} f \in \mathscr{D}(A)\right\}$ is a densely defined closed operator with adjoint $\left(-\pi_{1} A, \mathscr{D}\left(\pi_{1} A\right)\right)$. By von Neumann's theorem, see e.g. [18, Theorem 5.1.9], the operators $G:=\left(A \pi_{1}\right)^{*} A \pi_{1}$ and $I+\left(A \pi_{1}\right)^{*} A \pi_{1}$ with domain

$$
\mathscr{D}(G):=\mathscr{D}\left(\left(A \pi_{1}\right)^{*}\left(A \pi_{1}\right)\right)=\left\{f \in \mathscr{D}\left(A \pi_{1}\right): A \pi_{1} f \in \mathscr{D}\left(\left(A \pi_{1}\right)^{*}\right)\right\}
$$

are are self-adjoint. Furthermore, the latter one is injective and surjective (with range equal to $\mathbb{H}$ ) and admits a bounded linear inverse. We define the operator $B$ with domain $\mathscr{D}(B)=\mathscr{D}\left(\left(A \pi_{1}\right)^{*}\right)$ via

$$
B:=\left(I+\left(A \pi_{1}\right)^{*} A \pi_{1}\right)^{-1}\left(A \pi_{1}\right)^{*} .
$$


Then $B^{*}=A \pi_{1}\left(I+\left(A \pi_{1}\right)^{*} A \pi_{1}\right)^{-1}$ defined on $\mathscr{D}\left(B^{*}\right)=\mathbb{H}$ is closed and bounded. Consequently, $\left(B, D\left(\left(A \pi_{1}\right)^{*}\right)\right)$ is also bounded and has a unique extension to a bounded linear operator $(B, \mathbb{H})$. By e.g. [18, Theorem 5.1.9], we have

$$
B=\left(A \pi_{1}\right)^{*}\left(I+A \pi_{1}\left(A \pi_{1}\right)^{*}\right)^{-1} .
$$

Consequently, $\|B\| \leq 1$ and $\pi_{1} B=B$.

We shall need the following two more assumptions.

(H3) We assume $\mathscr{D} \subset \mathscr{D}(G)$. Furthermore, there exists a constant $N \geq 1$ such that

$$
\left\langle B S \pi_{2} f, \pi_{1} f\right\rangle \leq \frac{N}{2}\left\|\pi_{1} f\right\| \cdot\left\|\pi_{2} f\right\|, \quad-\left\langle B A \pi_{2} f, \pi_{1} f\right\rangle \leq \frac{N}{2}\left\|\pi_{1} f\right\| \cdot\left\|\pi_{2} f\right\|, \quad f \in \mathscr{D} .
$$

(H4) For any $f \in \mathscr{D}(L)$ there exists a sequence $\left\{f_{n}\right\}_{n \geq 1} \subset \mathscr{D}$ such that $f_{n} \rightarrow f$ in $\mathbb{H}$ and

$$
\limsup _{n \rightarrow \infty}\left\langle-L f_{n}, f_{n}\right\rangle \leq\langle-L f, f\rangle, \limsup _{n \rightarrow \infty} \Psi\left(f_{n}\right) \leq \Psi(f)
$$

Theorem 2.1. Assume (H1)-(H4) and let $\Psi$ satisfy

$$
\Psi\left(P_{t} f\right) \leq \Psi(f), \quad \Psi\left(\mathrm{e}^{-t G} f\right) \leq \Psi(f), \quad \Psi\left(\pi_{1} f\right) \leq \Psi(f), \quad f \in \mathbb{H} .
$$

If the weak Poincaré inequalities

$$
\left\|\pi_{1} f\right\|^{2} \leq \alpha_{1}(r)\left\|A \pi_{1} f\right\|^{2}+r \Psi\left(\pi_{1} f\right), \quad r>0, f \in \mathscr{D}\left(A \pi_{1}\right),
$$

and

$$
\left\|\pi_{2} f\right\|^{2} \leq \alpha_{2}(r)\langle-S f, f\rangle+r \Psi(f), \quad r>0, f \in \mathscr{D}
$$

hold for some decreasing functions $\alpha_{i}:(0, \infty) \rightarrow[1, \infty), i=1,2$, then there exist constants $c_{1}, c_{2}>0$ such that (2.1) holds for

$$
\xi(t):=c_{1} \inf \left\{r>0: c_{2} t \geq \alpha_{1}(r)^{2} \alpha_{2}\left(\frac{r}{\alpha_{1}(r)^{2}}\right) \log \frac{1}{r}\right\}
$$

which goes to 0 as $t \rightarrow \infty$.

\subsection{Preparations}

Lemma 2.2. Under (H1)-(H3), we have

$$
\begin{gathered}
\|B f\| \leq \frac{1}{2}\left\|\pi_{2} f\right\|, \quad f \in \mathbb{H}, \\
\|A B f\| \leq\left\|\pi_{2} f\right\|, \quad f \in \mathscr{D}, \\
|\langle B f, L f\rangle| \leq\left\|\pi_{2} f\right\| \cdot\|f\|, \quad f \in \mathscr{D}(L), \\
\langle B L f, f\rangle \leq N\left\|\pi_{1} f\right\| \cdot\left\|\pi_{2} f\right\|-\left\langle(1+G)^{-1} G \pi_{1} f, \pi_{1} f\right\rangle, \quad f \in \mathscr{D}(L) .
\end{gathered}
$$


Proof. Let $f \in \mathscr{D}$ and $g=B f$. By (2.2), $\pi_{1} A^{*} \pi_{1} f=-\pi_{1} A \pi_{1} f=0$ and $\pi_{2} f \in \mathscr{D}(A)$ (see $(H 2)$ ), we have

$$
\begin{gathered}
\|g\|^{2}+\left\|A \pi_{1} g\right\|^{2}=\left\langle g+\left(A \pi_{1}\right)^{*} A \pi_{1} g, g\right\rangle=\left\langle\left(A \pi_{1}\right)^{*} f, g\right\rangle \\
=\left\langle\left(A \pi_{1}\right)^{*} \pi_{2} f, g\right\rangle=\left\langle\pi_{2} f, A \pi_{1} g\right\rangle \leq\left\|\pi_{2} f\right\| \cdot\left\|A \pi_{1} g\right\| .
\end{gathered}
$$

Combining this with

$$
\left\|\pi_{2} f\right\| \cdot\left\|A \pi_{1} g\right\| \leq \frac{1}{4}\left\|\pi_{2} f\right\|^{2}+\left\|A \pi_{1} g\right\|^{2}
$$

we obtain (2.7) for $f \in \mathscr{D}$, and hence for all $f \in \mathbb{H}$ since $\mathscr{D}$ is dense in $\mathbb{H}$ and the operators $B, \pi_{2}$ are bounded.

Next, combining (2.11) with $\pi_{1} B=B$ and

$$
\left\|\pi_{2} f\right\| \cdot\left\|A \pi_{1} g\right\| \leq \frac{1}{2}\left\|\pi_{2} f\right\|^{2}+\frac{1}{2}\left\|A \pi_{1} g\right\|^{2}
$$

we obtain

$$
\|A B f\|^{2}=\left\|A \pi_{1} B f\right\|^{2}=\left\|A \pi_{1} g\right\|^{2} \leq\left\|\pi_{2} f\right\|^{2}, \quad f \in \mathscr{D},
$$

which is equivalent to $(2.8)$.

Moreover, by the symmetry of $S$, antisymmetry of $A, S \pi_{1}=0$, and $B=\pi_{1} B$, we obtain from (2.8) that for any $f \in \mathscr{D}$,

$$
|\langle B f, L f\rangle|=|\langle B f,-A f\rangle|=|\langle A B f, f\rangle| \leq\left\|\pi_{2} f\right\| \cdot\|f\| .
$$

Since $\mathscr{D}$ is dense in $\mathscr{D}(L)$ and $B$ is bounded, this implies (2.9).

Finally, by $\pi_{1} B=B, S \pi_{1}=0$, the definition of $B$ and $(H 3)$, for $f \in \mathscr{D}$ we have

$$
\begin{aligned}
& \langle B L f, f\rangle=\left\langle B L f, \pi_{1} f\right\rangle=\left\langle B S f, \pi_{1} f\right\rangle-\left\langle B A f, \pi_{1} f\right\rangle \\
& =\left\langle B S \pi_{2} f, \pi_{1} f\right\rangle \\
-\left\langle B A \pi_{1} f, \pi_{1} f\right\rangle-\left\langle B A \pi_{2} f, \pi_{1} f\right\rangle & \\
& \leq N\left\|\pi_{1} f\right\| \cdot\left\|\pi_{2} f\right\|-\left\langle(1+G)^{-1} G \pi_{1} f, \pi_{1} f\right\rangle .
\end{aligned}
$$

By the boundedness of $(1+G)^{-1} G$ and that $\mathscr{D}$ is dense in $\mathscr{D}(L)$, this implies $(2.10)$.

Next, we need the following result on weak Poincaré inequality for subordinated operators. Let $\nu$ be a Lévy measure on $[0, \infty)$ such that $\int_{0}^{\infty}(r \wedge 1) \nu(\mathrm{d} r)<\infty$, then

$$
\phi_{\nu}(s):=\int_{0}^{\infty}\left(1-\mathrm{e}^{-s r}\right) \nu(\mathrm{d} r), \quad s \geq 0
$$

is a Bernstein function. Let $\left(S_{0}, \mathscr{D}\left(S_{0}\right)\right)$ be a non-negative definite self-adjoint operator. We intend to establish the weak Poincaré inequality for the form $\left\langle\phi_{\nu}\left(S_{0}\right) f, f\right\rangle$ in terms of that for $\left\langle S_{0} f, f\right\rangle$. The Nash type and super Poincaré inequalities have already been investigated in $[2,21]$. Recently, sub-exponential decay for subordinated semigroups was studied in [6], where $\phi_{\nu}$ is assumed to satisfy

$$
\liminf _{s \rightarrow \infty} \frac{\phi_{\nu}(s)}{\log s}>0
$$

However, this condition excludes $\phi_{\nu}(s):=\frac{s}{1+s}$ which is indeed what we need in the proof of Theorem 2.1. 
Lemma 2.3. Let $\left(A_{0}, \mathscr{D}\left(A_{0}\right)\right)$ be a densely defined closed linear operator on a separable Hilbert space $\mathbb{H}_{0}$. Let $P_{t}^{0}$ be the $C_{0}$-contraction semigroup generated by the self-adjoint operator $-A_{0}^{*} A_{0}$ with domain $\mathscr{D}\left(A_{0}^{*} A_{0}\right):=\left\{f \in \mathscr{D}\left(A_{0}\right): A_{0} f \in \mathscr{D}\left(A_{0}^{*}\right)\right\}$. If the weak Poincaré inequality

$$
\|f\|^{2} \leq \alpha(r)\left\|A_{0} f\right\|^{2}+r \Psi_{0}(f), \quad r>0, f \in \mathscr{D}\left(A_{0}\right)
$$

holds for some decreasing $\alpha:(0, \infty) \rightarrow(0, \infty)$, where $\Psi_{0}: \mathbb{H}_{0} \rightarrow[0, \infty]$ satisfies

$$
\Psi_{0}\left(P_{t}^{0} f\right) \leq \Psi_{0}(f), \quad t \geq 0, f \in \mathscr{D}\left(A_{0}\right),
$$

then

$$
\|f\|^{2} \leq\left(\int_{0}^{\infty}\left(1-\mathrm{e}^{-\frac{s}{\alpha(r)}}\right) \nu(\mathrm{d} s)\right)^{-1}\left\|\left(\phi_{\nu}\left(A_{0}^{*} A_{0}\right)\right)^{1 / 2} f\right\|^{2}+r \Psi(f), \quad r>0, f \in \mathscr{D}\left(A_{0}\right) .
$$

In particular, for $\nu(\mathrm{d} s)=\mathrm{e}^{-s} \mathrm{~d} s$ such that $\phi_{\nu}(s)=\frac{s}{1+s}$, we have

$$
\|f\|^{2} \leq(1+\alpha(r))\left\langle\left(1+A_{0}^{*} A_{0}\right)^{-1} A_{0}^{*} A_{0} f, f\right\rangle+r \Psi(f), \quad r>0, f \in \mathscr{D}\left(A_{0}\right) .
$$

Proof. Since $\mathscr{D}\left(\left(A_{0}^{*} A_{0}\right)^{1 / 2}\right)=\mathscr{D}\left(A_{0}\right)$, we have $\mathscr{D}\left(\left\{\phi_{\nu}\left(A_{0}^{*} A_{0}\right)\right\}^{1 / 2}\right) \supset \mathscr{D}\left(A_{0}\right)$. By (2.12) and (2.13), for any $f \in \mathscr{D}\left(A_{0}\right)$,

$$
\frac{\mathrm{d}}{\mathrm{d} t}\left\|P_{t}^{0} f\right\|^{2}=-2\left\|A_{0} P_{t}^{0} f\right\|^{2} \leq-\frac{2}{\alpha(r)}\left\|P_{t}^{0} f\right\|^{2}+\frac{2 r}{\alpha(r)} \Psi(f), \quad t \geq 0, r>0,
$$

because $P_{t}^{0}$ leaves $\mathscr{D}\left(A_{0}\right)$ invariant. Then Gronwall's lemma gives

$$
\left\|P_{t}^{0} f\right\|^{2} \leq \mathrm{e}^{-\frac{2 t}{\alpha(r)}}\|f\|^{2}+r \Psi(f)\left(1-\mathrm{e}^{-\frac{2 t}{\alpha(r)}}\right), \quad r>0, t \geq 0 .
$$

Therefore,

$$
\begin{aligned}
& \left\|\left(\phi_{\nu}\left(A_{0}^{*} A_{0}\right)\right)^{1 / 2} f\right\|^{2}=\int_{0}^{\infty}\left\langle f-P_{s}^{0} f, f\right\rangle \nu(\mathrm{d} s)=\int_{0}^{\infty}\left(\|f\|^{2}-\left\|P_{s / 2}^{0} f\right\|^{2}\right) \nu(\mathrm{d} s) \\
& \geq \int_{0}^{\infty}\left(\|f\|^{2}-\mathrm{e}^{-\frac{s}{\alpha(r)}}\|f\|^{2}-r \Psi(f)\left(1-\mathrm{e}^{-\frac{s}{\alpha(r)}}\right)\right) \nu(\mathrm{d} s) \\
& =\left(\|f\|^{2}-r \Psi(r)\right) \int_{0}^{\infty}\left(1-\mathrm{e}^{-\frac{s}{\alpha(r)}}\right) \nu(\mathrm{d} s), \quad r>0 .
\end{aligned}
$$

This implies the desired inequality.

In the proof of Theorem 1.1 (see Section 3 below), to verify (H3) we check the following two inequalities:

$$
\begin{aligned}
& \left\langle B S \pi_{2} f, \pi_{1} f\right\rangle \leq N\left\|\pi_{1} f\right\| \cdot\left\|\pi_{2} f\right\|, \\
& \left\langle B A \pi_{2} f, \pi_{1} f\right\rangle \leq N\left\|\pi_{1} f\right\| \cdot\left\|\pi_{2} f\right\|, \quad f \in \mathscr{D} .
\end{aligned}
$$

The first inequality is easy to check there, see Section 3, the first part in the proof of (H3). To verify the second, we present below a sufficient condition provided in [12, Prop. 2.15]. 
Proposition 2.4. Assume that $(-G, \mathscr{D})$ is essentially $m$-dissipative (equivalently, essentially self-adjoint). If there exists constant $N \in(0, \infty)$ such that

$$
\left\|(B A)^{*} g\right\| \leq N\|g\| \quad \text { for all } g=(I+G) f, f \in \mathscr{D},
$$

then

$$
\left|\left\langle B A \pi_{2} f, \pi_{1} f\right\rangle\right| \leq N\left\|\pi_{1} f\right\| \cdot\left\|\pi_{2} f\right\|, \quad f \in \mathscr{D} .
$$

\subsection{Proof of Theorem 2.1}

Proof. For any $\varepsilon \in[0,1)$, let

$$
I_{\varepsilon}(f)=\frac{1}{2}\|f\|^{2}+\varepsilon\langle B f, f\rangle, \quad f \in \mathbb{H} .
$$

By (2.7), we have

$$
\frac{1-\varepsilon}{2}\|f\|^{2} \leq I_{\varepsilon}(f) \leq \frac{1+\varepsilon}{2}\|f\|^{2}, \quad f \in \mathbb{H}
$$

Now, let $f \in \mathscr{D}$ and $f_{t}=P_{t} f$ for $t \geq 0$. We have

$$
\frac{\mathrm{d}}{\mathrm{d} t} I_{\varepsilon}\left(f_{t}\right)=\left\langle L f_{t}, f_{t}\right\rangle+\varepsilon\left\langle B L f_{t}, f_{t}\right\rangle+\varepsilon\left\langle B f_{t}, L f_{t}\right\rangle .
$$

By $(2.5)$ and $\langle-L g, g\rangle=\langle-S g, g\rangle$ for $g \in \mathscr{D}$, we obtain

$$
\langle L g, g\rangle \leq-\frac{\left\|\pi_{2} g\right\|^{2}}{\alpha_{2}\left(r_{2}\right)}+\frac{r_{2} \Psi(g)}{\alpha_{2}\left(r_{2}\right)}, \quad g \in \mathscr{D}, r_{2}>0
$$

Since $f_{t} \in \mathscr{D}(L)$, combining this with $(H 4)$ and (2.3), we arrive at

$$
\left\langle L f_{t}, f_{t}\right\rangle \leq-\frac{\left\|\pi_{2} f_{t}\right\|^{2}}{\alpha_{2}\left(r_{2}\right)}+\frac{r_{2} \Psi\left(f_{t}\right)}{\alpha_{2}\left(r_{2}\right)} \leq-\frac{\left\|\pi_{2} f_{t}\right\|^{2}}{\alpha_{2}\left(r_{2}\right)}+\frac{r_{2} \Psi(f)}{\alpha_{2}\left(r_{2}\right)}, \quad t, r_{2}>0 .
$$

Next, applying Lemma 2.3 with $\mathbb{H}_{0}=\mathbb{H}_{1}, A_{0}=\left.\left(\left(A \pi_{1}\right)^{*}\left(A \pi_{1}\right)\right)^{1 / 2}\right|_{\mathbb{H}_{1}}$ and $\Psi_{0}=\left.\Psi\right|_{\mathbb{H}_{1}}$ such that condition (2.13) follows from (2.3), we see that (2.4) implies

$$
-\left\langle\left(I+\left(A \pi_{1}\right)^{*}\left(A \pi_{1}\right)\right)^{-1}\left(A \pi_{1}\right)^{*} A \pi_{1} f, \pi_{1} f\right\rangle \leq-\frac{\left\|\pi_{1} f\right\|^{2}}{\alpha_{1}\left(r_{1}\right)+1}+\frac{r_{1} \Psi\left(\pi_{1} f\right)}{\alpha_{1}\left(r_{1}\right)+1}, \quad r>0, f \in \mathscr{D}\left(A \pi_{1}\right) .
$$

Since the operator $\left(I+\left(A \pi_{1}\right)^{*}\left(A \pi_{1}\right)\right)^{-1}\left(A \pi_{1}\right)^{*} A \pi_{1}$ is bounded, $\mathscr{D}\left(A \pi_{1}\right) \supset \mathscr{D}$ due to $(H 2)$, and by $(H 4)$ for any $g \in \mathscr{D}(L)$ we may find a sequence $g_{n} \in \mathscr{D}$ such that $g_{n} \rightarrow g$ in $\mathbb{H}$ and $\limsup _{n \rightarrow \infty} \Psi\left(g_{n}\right) \leq \Psi(g)$, this inequality holds for all $g \in \mathscr{D}(L)$. Combining this with (2.10) and (2.3), we obtain

$$
\begin{aligned}
\left\langle B L f_{t}, f_{t}\right\rangle & \leq N\left\|\pi_{1} f_{t}\right\| \cdot\left\|\pi_{2} f_{t}\right\|-\left\langle\left(I+\left(A \pi_{1}\right)^{*}\left(A \pi_{1}\right)\right)^{-1}\left(A \pi_{1}\right)^{*} A \pi_{1} f_{t}, \pi_{1} f_{t}\right\rangle \\
& \leq N\left\|\pi_{1} f_{t}\right\| \cdot\left\|\pi_{2} f_{t}\right\|-\frac{\left\|\pi_{1} f_{t}\right\|^{2}}{\alpha_{1}\left(r_{1}\right)+1}+\frac{r_{1} \Psi(f)}{\alpha_{1}\left(r_{1}\right)+1}, \quad t, r_{1}>0 .
\end{aligned}
$$


Substituting (2.9), (2.19) and (2.20) into (2.18), we arrive at

$$
\begin{aligned}
\frac{\mathrm{d}}{\mathrm{d} t} I_{\varepsilon}\left(f_{t}\right) \leq & -\left(\frac{\left\|\pi_{2} f_{t}\right\|^{2}}{\alpha_{2}\left(r_{2}\right)}+\frac{\varepsilon\left\|\pi_{1} f_{t}\right\|^{2}}{\alpha_{1}\left(r_{1}\right)+1}\right)+\varepsilon\left(N\left\|\pi_{1} f_{t}\right\| \cdot\left\|\pi_{2} f_{t}\right\|+\left\|\pi_{2} f_{t}\right\| \cdot\left\|f_{t}\right\|\right) \\
& +\Psi(f)\left(\frac{r_{2}}{\alpha_{2}\left(r_{2}\right)}+\frac{\varepsilon r_{1}}{\alpha_{1}\left(r_{1}\right)+1}\right), \quad t \geq 0, f \in \mathscr{D} .
\end{aligned}
$$

Combining this with

$$
\begin{aligned}
& \varepsilon N\left\|\pi_{1} f_{t}\right\| \cdot\left\|\pi_{2} f_{t}\right\| \leq \frac{\varepsilon\left\|\pi_{1} f_{t}\right\|^{2}}{2\left(\alpha_{1}\left(r_{1}\right)+1\right)}+\frac{\varepsilon N^{2}\left(\alpha_{1}\left(r_{1}\right)+1\right)\left\|\pi_{2} f_{t}\right\|^{2}}{2}, \\
& \varepsilon\left\|\pi_{2} f_{t}\right\| \cdot\left\|f_{t}\right\| \leq \frac{\left\|\pi_{2} f_{t}\right\|^{2}}{2 \alpha_{2}\left(r_{2}\right)}+\frac{\varepsilon^{2} \alpha_{2}\left(r_{2}\right)\left\|f_{t}\right\|^{2}}{2}
\end{aligned}
$$

we obtain

$$
\begin{aligned}
\frac{\mathrm{d}}{\mathrm{d} t} I_{\varepsilon}\left(f_{t}\right) \leq & -\left(\frac{1}{2 \alpha_{2}\left(r_{2}\right)}-\frac{\varepsilon N^{2}\left(\alpha_{1}\left(r_{1}\right)+1\right)}{2}\right)\left\|\pi_{2} f_{t}\right\|^{2}-\frac{\varepsilon\left\|\pi_{1} f_{t}\right\|^{2}}{2\left(\alpha_{1}\left(r_{1}\right)+1\right)} \\
& +\frac{\varepsilon^{2} \alpha_{2}\left(r_{2}\right)\left\|f_{t}\right\|^{2}}{2}+\Psi(f)\left(\frac{r_{2}}{\alpha_{2}\left(r_{2}\right)}+\frac{\varepsilon r_{1}}{\alpha_{1}\left(r_{1}\right)+1}\right), \quad t \geq 0, f \in \mathscr{D} .
\end{aligned}
$$

Taking

$$
\varepsilon=\frac{1}{2 N^{2}\left(\alpha_{1}\left(r_{1}\right)+1\right) \alpha_{2}\left(r_{2}\right)} \leq \frac{1}{2}
$$

since $N, \alpha_{2} \geq 1$, we have

$$
\begin{aligned}
& \frac{1}{2 \alpha_{2}\left(r_{2}\right)}-\frac{\varepsilon N^{2}\left(\alpha_{1}\left(r_{1}\right)+1\right)}{2} \geq \frac{1}{4 \alpha_{2}\left(r_{2}\right)}, \\
& \frac{1}{4 \alpha_{2}\left(r_{2}\right)} \wedge \frac{\varepsilon}{2\left(\alpha_{1}\left(r_{1}\right)+1\right)} \geq \varepsilon^{2} \alpha_{2}\left(r_{2}\right) .
\end{aligned}
$$

Then (2.21) implies

$$
\frac{\mathrm{d}}{\mathrm{d} t} I_{\varepsilon}\left(f_{t}\right) \leq-\frac{\left\|f_{t}\right\|^{2}}{8 N^{4} \alpha_{2}\left(r_{2}\right)\left(\alpha_{1}\left(r_{1}\right)+1\right)^{2}}+\Psi(f)\left(\frac{r_{2}}{\alpha_{2}\left(r_{2}\right)}+\frac{r_{1}}{2 N^{2} \alpha_{2}\left(r_{2}\right)\left(\alpha_{1}\left(r_{1}\right)+1\right)^{2}}\right) .
$$

Since $\varepsilon \leq \frac{1}{2}$, by (2.17) we have $\left\|f_{t}\right\|^{2} \geq \frac{4}{3} I_{\varepsilon}\left(f_{t}\right)$, so that

$$
\frac{\mathrm{d}}{\mathrm{d} t} I_{\varepsilon}\left(f_{t}\right) \leq-\frac{I_{\varepsilon}\left(f_{t}\right)}{6 N^{4} \alpha_{2}\left(r_{2}\right)\left(\alpha_{1}\left(r_{1}\right)+1\right)^{2}}+\Psi(f)\left(\frac{r_{2}}{\alpha_{2}\left(r_{2}\right)}+\frac{r_{1}}{2 N^{2} \alpha_{2}\left(r_{2}\right)\left(\alpha_{1}\left(r_{1}\right)+1\right)^{2}}\right) .
$$

By Gronwall's lemma and (2.22), we arrive at

$$
I_{\varepsilon}\left(f_{t}\right) \leq \exp \left[-\frac{t}{6 N^{4} \alpha_{2}\left(r_{2}\right)\left(\alpha_{1}\left(r_{1}\right)+1\right)^{2}}\right] I_{\varepsilon}(f)+\Psi(f)\left(3 N^{2} r_{1}+6 N^{4} r_{2}\left(\alpha_{1}\left(r_{1}\right)+1\right)^{2}\right) .
$$


Taking $r_{1}=r, r_{2}=\frac{r}{\alpha_{1}(r)^{2}}$, using $(2.17)$ for $\varepsilon \in\left(0, \frac{1}{2}\right)$ and that $\alpha_{1}(r) \geq 1$, obtain

$$
\left\|f_{t}\right\|^{2} \leq c_{1} \exp \left[-\frac{c_{2} t}{\alpha_{1}(r)^{2} \alpha_{2}\left(\frac{r}{\alpha_{1}(r)^{2}}\right)}\right]\|f\|^{2}+c_{1} r \Psi(f), \quad r>0, f \in \mathscr{D}, t \geq 0 .
$$

Consequently, for any $r>0$ such that $c_{2} t \geq \alpha_{1}(r)^{2} \alpha_{2}\left(\frac{r}{\alpha_{1}(r)^{2}}\right) \log \frac{1}{r}$, we have

$$
\left\|f_{t}\right\|^{2} \leq c_{1} r\left(\|f\|^{2}+\Psi(f)\right) .
$$

Therefore, (2.1) with $\xi(t)$ in (2.6) holds for $f \in \mathscr{D}$. By $(H 4)$, it holds for all $f \in \mathscr{D}(L)$. Then the proof is finished.

\section{Proof of Theorem 1.1}

We first embed $P_{t}$ in the framework of Section 2. Since $\sigma$ is invertible, we have $\sigma y-b=$ $\sigma\left(y-\sigma^{-1} b\right)$. So, with the shift $y \mapsto y+\sigma^{-1} b$ for the second variable $y$, in $(H)$ we may and do take $b=0$, i.e. $V_{2}(y)=\Phi\left(|\sigma y|^{2}\right)$. Since we may move $\sigma$ from the potential $V_{2}$ to the symmetric part of the generator $L$ corresponding to the solution of (1.9) and the matrix $Q$ as described in Remark 1.1(2), we only have to consider the case $V_{2}(y)=\Phi\left(|y|^{2}\right)$. Thus

$$
\nabla^{(2)} V_{2}(y)=2 \Phi^{\prime}\left(|y|^{2}\right) y
$$

Let

$$
\mu=\mu_{1} \times \mu_{2}, \quad \text { where } \mu_{i}\left(\mathrm{~d} x_{i}\right):=Z\left(V_{i}\right)^{-1} \mathrm{e}^{-V_{i}\left(x_{i}\right)} \mathrm{d} x_{i} \text { on } \mathbb{R}^{d_{1}}, i=1,2 .
$$

By Itô's formula, the generator $L$ for the solution to (1.9) has the decomposition

$$
L=S-A
$$

where

$$
\begin{aligned}
S & :=\Delta^{(2)}-\left\langle\left(\nabla^{(2)} V_{2}\right), \nabla^{(2)} \cdot\right\rangle=\sum_{i=1}^{d_{2}}\left(\partial_{y_{i}}^{2}-\left(\partial_{y_{i}} V_{2}\right) \partial_{y_{i}}\right) \\
A & :=\left\langle Q^{*}\left(\nabla^{(1)} V_{1}\right), \nabla^{(2)} \cdot\right\rangle-\left\langle Q\left(\nabla^{(2)} V_{2}\right), \nabla^{(1)} \cdot\right\rangle \\
& =\sum_{i=1}^{d_{1}} \sum_{j=1}^{d_{2}} Q_{i j}\left(\left(\partial_{x_{i}} V_{1}\right) \partial_{y_{j}}-\left(\partial_{y_{j}} V_{2}\right) \partial_{x_{i}}\right) .
\end{aligned}
$$

Since above we moved $\sigma$ from the potential $V_{2}$ to the symmetric part of $L$ and to the matrix $Q$, instead of $S$ and $Q$ we should consider

$$
\sum_{i, j=1}^{d_{2}}\left(\sigma \sigma^{*}\right)_{i j}\left(\partial_{y_{i}} \partial_{y_{j}}-\left(\partial_{y_{i}} V_{2}\right) \partial_{y_{j}}\right) \text { and } Q \sigma^{*}
$$


respectively. But, because $\sigma \sigma^{*}$ is a constant, symmetric, invertible matrix, without loss of generality we may take $\sigma$ equal to the identity matrix. The considerations below easily generalize to general $\sigma$, but are easier to follow for $\sigma$ being the identity matrix.

Let $\nabla=\left(\nabla^{(1)}, \nabla^{(2)}\right)$ be the gradient operator on $\mathbb{R}^{d_{1}+d_{2}}$, and denote

$$
C_{c}^{\infty}\left(\mathbb{R}^{d_{1}+d_{2}}\right)=\left\{f \in C^{\infty}\left(\mathbb{R}^{d_{1}+d_{2}}\right): \nabla f \text { has compact support }\right\} .
$$

The integration by parts formula implies that $\left(S, C_{c}^{\infty}\left(\mathbb{R}^{d_{1}+d_{2}}\right)\right)$ is symmetric and non-positive definite in $L^{2}(\mu)$ while $\left(A, C_{c}^{\infty}\left(\mathbb{R}^{d_{1}+d_{2}}\right)\right)$ is antisymmetric in $L^{2}(\mu)$. Consequently, $L^{*}:=$ $L+2 A=S+A$ satisfies

$$
\mu(f L g)=\mu\left(g L^{*} f\right), \quad f, g \in C_{c}^{\infty}\left(\mathbb{R}^{d_{1}+d_{2}}\right) .
$$

Therefore, $\left(L, C_{c}^{\infty}\left(\mathbb{R}^{d_{1}+d_{2}}\right)\right.$ is dissipative and, in particular, closable in $L^{2}(\mu)$. Let $(L, \mathscr{D}(L))$ denote the closure. For our analysis, however, we need more than closability. We need that the closure $(L, \mathscr{D}(L))$ is m-dissipative, i.e. the operator $(L, \mathscr{D}(L))$ is dissipative and the operator $(I-L): \mathscr{D}(L)) \rightarrow L^{2}(\mu)$ is surjective. This is implied by $\left(L, C_{c}^{\infty}\left(\mathbb{R}^{d_{1}+d_{2}}\right)\right.$ being essentially m-dissipative, i.e. $\left(L, C_{c}^{\infty}\left(\mathbb{R}^{d_{1}+d_{2}}\right)\right.$ is dissipative and $(I-L)(\mathscr{D}(L)) \subset L^{2}(\mu)$ is dense. For a densely defined operator being $\mathrm{m}$-dissipative is equivalent to be the generator of a $C_{0}$-contraction semigroup. Essential m-dissipativity is a uniqueness result. It implies that the generator of the semigroup is uniquely determined on a given dense set of nice functions. This is of crucial importance for the present approach, because the conditions of Theorem 2.1 usually can only be checked on nice functions. Essential m-dissipativity is also a useful tool to related a $C_{0}$-contraction semigroup uniquely to the solution of an SDE. This, and moreover the first assertion of Theorem 1.1, we show in the following proposition.

Proposition 3.1. Under assumption $(H)$, the operator $\left(L, C_{c}^{\infty}\left(\mathbb{R}^{d_{1}+d_{2}}\right)\right)$ is essentially $m$ dissipative in $L^{2}(\mu)$, and the $C_{0}$-contraction semigroup $T_{t}$ generated by the closure coincides with $P_{t}$ in $L^{2}(\mu)$. Consequently, the solution to (1.9) is non-explosive and $\mu$ is an invariant probability measure of $P_{t}$.

Proof. In [17, Theorem 3.10] under even weaker assumptions as in $(H)$, essential m-dissipativity of $\left(L, C_{c}^{\infty}\left(\mathbb{R}^{d_{1}+d_{2}}\right)\right)$ in $L^{2}(\mu)$ is shown. In the proof condition (1.10) for $i=2$ is used. Hence the closure $(L, \mathscr{D}(L))$ generates a $C_{0}$-contraction semigroup $T_{t}$. Then $\mu(L f)=0$ for $f \in \mathscr{D}(L)$ implies that

$$
\partial_{t} \mu\left(T_{t} f\right)=\mu\left(L T_{t} f\right)=0, \quad t \geq 0, f \in \mathscr{D}(L)
$$

so that $\mu$ is an invariant probability measure of $T_{t}$. On the other hand, according to $[3$, Theorem 1.1 and Proposition 1.4] (see also [5, Theorem 3.17 and Remark 3.18]), for $\mu$ a.e. starting point $z=(x, y) \in \mathbb{R}^{d_{1}+d_{2}}$ there is a law $\mathbb{P}^{z}$ on the space of $\mathbb{R}^{d_{1}+d_{2}}$-valued continuous functions such that $\left(X_{t}, Y_{t}\right)_{t \geq 0}$ is a weak solution to (1.9) and for any distribution $\nu(\mathrm{d} z)=\rho(z) \mu(\mathrm{d} z)$ with a probability density $\rho$

$$
\mu\left(\rho T_{t} f\right)=\int_{\mathbb{R}^{d_{1}+d_{2}}} \mathbb{E}^{z}\left[f\left(X_{t}, Y_{t}\right)\right] \nu(d z), \quad t \geq 0, f \in \mathscr{B}_{b}\left(\mathbb{R}^{d_{1}+d_{2}}\right) .
$$


By the uniqueness of the $\operatorname{SDE}(1.9)$, we have for $\mu$-a.e. $z \in \mathbb{R}^{d_{1}+d_{2}}$ :

$$
P_{t} f(x, y)=\mathbb{E}^{z}\left[f\left(X_{t}, Y_{t}\right)\right], \quad t \geq 0, f \in \mathscr{B}_{b}\left(\mathbb{R}^{d_{1}+d_{2}}\right) .
$$

Therefore, $\mu\left(\rho P_{t} f\right)=\mu\left(\rho T_{t} f\right)$ holds for any $\rho \in L^{1}(\mu), t \geq 0$ and $f \in \mathscr{B}_{b}\left(\mathbb{R}^{d_{1}+d_{2}}\right)$, and hence, $P_{t}$ is a $\mu$-version of $T_{t}$. Consequently, $\mu$ is an invariant probability measure of $P_{t}$. Since $P_{t} 1 \leq 1$, this implies that $P_{t} 1=1, \mu$-a.e. Since the coefficients of the SDE is at least $C^{1}$-smooth, the semigroup $P_{t}$ is Feller so that $P_{t} 1$ is continuous. Therefore, $P_{t} 1(z)=1$ holds for all $z \in \mathbb{R}^{d_{1}+d_{2}}$, i.e. the solution to (1.9) is non-explosive.

Now, to prove the second assertion in Theorem 1.1 using Theorem 2.1, we take

$$
\mathbb{H}=\left\{f \in L^{2}(\mu): \mu(f)=0\right\}, \mathbb{H}_{1}=\{f \in \mathbb{H}: f(x, y) \text { does not depend on } y\} .
$$

Then

$$
\left(\pi_{1} f\right)(x, y)=\pi_{1} f(x):=\int_{\mathbb{R}^{d_{2}}} f(x, y) \mu_{2}(\mathrm{~d} y), \quad f \in \mathbb{H} .
$$

Let

$$
\mathscr{D}=\mathbb{H} \cap C_{c}^{\infty}\left(\mathbb{R}^{d_{1}+d_{2}}\right)=\left\{f \in C_{c}^{\infty}\left(\mathbb{R}^{d_{1}+d_{2}}\right): \mu(f)=0\right\} .
$$

Let $(L, \mathscr{D}(L)),(S, \mathscr{D}(S))$ and $(A, \mathscr{D}(A))$ be the closures in $\mathbb{H}$ of $(L, \mathscr{D}),(S, \mathscr{D})$ and $(A, \mathscr{D})$ respectively. Since the closure of $\left(L, C_{c}^{\infty}\left(\mathbb{R}^{d_{1}+d_{2}}\right)\right)$ in $L^{2}(\mu)$ generates a strongly continuous contraction semigroup, see Proposition 3.1, we have $L^{2}(\mu)=\overline{\mathcal{R}(L)} \oplus \mathcal{N}(L)$, see [14, Theorem 8.20]. Hence, because the constant functions are in $\mathcal{N}(L)$, the operator $(L, \mathscr{D})$ is essentially m-dissipative in $\mathbb{H}$.

We verify assumptions $(H 1)-(H 4)$ as follows.

Proof of $(H 1):$ Let $f \in \mathbb{H}$. Then $\pi_{1} f \in L^{2}\left(\mu_{1}\right)$ with $\mu_{1}\left(\pi_{1} f\right)=0$. Let $\left\{g_{n}\right\}_{n \geq 0} \subset$ $C_{c}^{\infty}\left(\mathbb{R}^{d_{1}}\right)$ such that $\mu_{1}\left(g_{n}\right)=0$ and $\mu_{1}\left(\left|g_{n}-\pi_{1} f\right|^{2}\right) \rightarrow 0$. Let $\tilde{g}_{n}(x, y)=g_{n}(x)$. Then $\tilde{g}_{n} \in \mathscr{D}$, $\mu\left(\left|\tilde{g}_{n}-\pi_{1} f\right|^{2}\right)=\mu_{1}\left(\left|g_{n}-\pi_{1} f\right|^{2}\right) \rightarrow 0$ and

$$
\lim _{n, m \rightarrow \infty} \mu\left(\left|\tilde{g}_{n}-\tilde{g}_{m}\right|^{2}+\left|S \tilde{g}_{n}-S \tilde{g}_{m}\right|^{2}\right)=\lim _{n, m \rightarrow \infty} \mu\left(\left|\tilde{g}_{n}-\tilde{g}_{m}\right|^{2}\right)=0 .
$$

Thus, $\left\{\tilde{g}_{n}\right\}_{n \geq 1}$ is a Cauchy sequence in $\mathscr{D}(S)$ with $S g_{n}=0$, and converges to $\pi_{1} f$ in $L^{2}(\mu)$. Therefore, $\pi_{1} f \in \mathscr{D}(S)$ and $S \pi_{1} f=0$ since the operator is closed.

Proof of $(H 2): \quad$ For any $f \in \mathscr{D}$, we have $\pi_{1} f \in \mathscr{D}$ depending only on the first component. So, $\pi_{1} \mathscr{D} \subset \mathscr{D} \subset \mathscr{D}(A)$. Since $\left(\pi_{1} f\right)(x, y)=\pi_{1} f(x)$ only depends on $x$, by the definitions of $A$ and $\pi_{1}$, we have

$$
-\left(\pi_{1} A \pi_{1}\right) f(x, y)=\int_{\mathbb{R}^{d_{2}}}\left\langle Q \nabla^{(2)} V_{2}\left(y^{\prime}\right), \nabla^{(1)} \pi_{1} f(x)\right\rangle \mu_{2}\left(\mathrm{~d} y^{\prime}\right)=\left\langle\mu_{2}\left(Q \nabla^{(2)} V_{2}\right), \nabla^{(1)} \pi_{1} f(x)\right\rangle=0,
$$

where the last step is due to $V_{2}(y)=\Phi\left(|y|^{2}\right)$ and $\left|\nabla V_{2}\right| \in L^{1}\left(\mu_{2}\right)$ according to $(H)$. Then (H2) holds. 
Proof of $(H 3)$ : It suffices to prove (2.15). For the first inequality, we only need to find out a bounded measurable function $K$ such that

$$
S A \pi_{1} f=K A \pi_{1} f, \quad f \in \mathscr{D},
$$

since this implies

$$
\begin{aligned}
B S & =\left(I+\left(A \pi_{1}\right)^{*} A \pi_{1}\right)^{-1}\left(A \pi_{1}\right)^{*} S=\left(I+\left(A \pi_{1}\right)^{*} A \pi_{1}\right)^{-1}\left(S A \pi_{1}\right)^{*} \\
& =\left(I+\left(A \pi_{1}\right)^{*} A \pi_{1}\right)^{-1}\left(K A \pi_{1}\right)^{*}=B K,
\end{aligned}
$$

so that by $\|B\| \leq 1$ we have

$$
\left|\left\langle B S \pi_{2} f, \pi_{1} f\right\rangle\right|=\left|\left\langle B K \pi_{2} f, \pi_{1} f\right\rangle\right| \leq\|K\|_{\infty}\left\|\pi_{2} f\right\| \cdot\left\|\pi_{1} f\right\| .
$$

Now for any $f \in \mathscr{D},(3.1)$ implies

$$
\begin{aligned}
& \left(S A \pi_{1} f\right)(x, y)=S\left\langle Q \nabla^{(2)} V_{2}, \nabla^{(1)} \pi_{1} f\right\rangle(x, y) \\
& =\left(\Delta^{(2)}-\left\langle\nabla^{(2)} V_{2}, \nabla^{(2)} \cdot\right\rangle\right) \sum_{i=1}^{d_{1}}\left(2 \Phi^{\prime}\left(|y|^{2}\right)(Q y)_{i} \partial_{x_{i}} \pi_{1} f(x)\right) \\
& =2 \sum_{i=1}^{d_{1}}\left(\Phi^{\prime \prime}\left(|y|^{2}\right)\left(2 d_{2}-4 \Phi^{\prime}\left(|y|^{2}\right)|y|^{2}+4\right)-2 \Phi^{\prime}\left(|y|^{2}\right)^{2}+4 \Phi^{\prime \prime \prime}\left(|y|^{2}\right)|y|^{2}\right)(Q y)_{i} \partial_{x_{i}} \pi_{1} f(x) \\
& =2 H\left(|y|^{2}\right)\left\langle Q \nabla^{(2)} V_{2}(y), \nabla^{(1)} \pi_{1} f(x)\right\rangle=2 H\left(|y|^{2}\right)\left(A \pi_{1} f\right)(x, y),
\end{aligned}
$$

where

$$
H(r):=\frac{2 r \Phi^{\prime \prime \prime}(r)+\left(d_{2}+2\right) \Phi^{\prime \prime}(r)}{\Phi^{\prime}(r)}-\Phi^{\prime}(r)-2 r \Phi^{\prime \prime}(r), \quad r>0,
$$

is bounded according to $(H)$. Then (3.4) holds for some bounded function $K$.

To prove the second inequality in $(2.15)$, we consider the operator $G:=-\pi_{1} A^{2} \pi_{1}=$ $\left(A \pi_{1}\right)^{*} A \pi_{1}$ on $\mathscr{D}$. By the definitions of $A$ and $\pi_{1}$, we have

$$
\begin{aligned}
(G f)(x, y)=(G f)(x)= & \int_{\mathbb{R}^{d_{2}}}-\operatorname{Hess}_{\pi_{1} f}\left(Q \nabla^{(2)} V_{2}\left(y^{\prime}\right), Q \nabla^{(2)} V_{2}\left(y^{\prime}\right)\right)(x) \\
& \operatorname{Hess}_{V_{2}}\left(Q^{*} \nabla^{(1)} V_{1}(x), Q^{*} \nabla^{(1)} \pi_{1} f(x)\right)\left(y^{\prime}\right) \mu_{2}\left(\mathrm{~d} y^{\prime}\right) .
\end{aligned}
$$

Then (3.1) implies

$$
\begin{aligned}
& \int_{\mathbb{R}^{d_{2}}} \operatorname{Hess}_{\pi_{1} f}\left(Q \nabla^{(2)} V_{2}(y), Q \nabla^{(2)} V_{2}(y)\right)(x) \mu_{2}(\mathrm{~d} y) \\
& =4 \sum_{i, j=1}^{d_{1}} \int_{\mathbb{R}^{d_{2}}}\left(\partial_{x_{i}} \partial_{x_{j}} \pi_{1} f\right)(x) \Phi^{\prime}\left(|y|^{2}\right)^{2}(Q y)_{i}(Q y)_{j} \mu_{2}(\mathrm{~d} y) \\
& =4 \sum_{i, j=1}^{d_{1}} \sum_{k=1}^{d_{2}} \int_{\mathbb{R}^{d_{2}}}\left(\partial_{x_{i}} \partial_{x_{j}} \pi_{1} f\right)(x) \Phi^{\prime}\left(|y|^{2}\right)^{2} Q_{i k} Q_{i k} y_{k}^{2} \mu_{2}(\mathrm{~d} y)
\end{aligned}
$$




$$
\begin{aligned}
& =\frac{4}{d_{2}} \sum_{i, j=1}^{d_{1}} \int_{\mathbb{R}^{d_{2}}}\left(Q Q^{*}\right)_{i j}\left(\partial_{x_{i}} \partial_{x_{j}} \pi_{1} f\right)(x) \Phi^{\prime}\left(|y|^{2}\right)^{2}|y|^{2} \mu_{2}(\mathrm{~d} y) \\
& =\frac{\mu_{2}\left(\left|\nabla V_{2}\right|^{2}\right)}{d_{2}} \sum_{i, j=1}^{d_{1}}\left(Q Q^{*}\right)_{i j}\left(\partial_{x_{i}} \partial_{x_{j}} \pi_{1} f\right)(x) .
\end{aligned}
$$

Similarly,

$$
\begin{aligned}
& \int_{\mathbb{R}^{d_{2}}} \operatorname{Hess}_{V_{2}}\left(Q^{*} \nabla^{(1)} V_{1}(x), Q^{*} \nabla^{(1)} \pi_{1} f(x)\right)(y) \mu_{2}(\mathrm{~d} y) \\
& =\left\langle Q^{*} \nabla^{(1)} V_{1}(x), Q^{*} \nabla^{(1)} \pi_{1} f(x)\right\rangle \int_{\mathbb{R}^{d_{2}}} 2 \Phi^{\prime}\left(|y|^{2}\right)+\frac{4 \Phi^{\prime \prime}\left(|y|^{2}\right)|y|^{2}}{d_{2}} \mu_{2}(\mathrm{~d} y) \\
& =\frac{\left\langle Q^{*} \nabla^{(1)} V_{1}(x), Q^{*} \nabla^{(1)} \pi_{1} f(x)\right\rangle}{d_{2}} \int_{\mathbb{R}^{d_{2}}} \Delta^{(2)} V_{2}(y) \mu_{2}(\mathrm{~d} y) \\
& =\frac{\mu_{2}\left(\left|\nabla^{(2)} V_{2}\right|^{2}\right)}{d_{2}}\left\langle Q^{*} \nabla^{(1)} V_{1}(x), Q^{*} \nabla^{(1)} \pi_{1} f(x)\right\rangle .
\end{aligned}
$$

Therefore, letting $N\left(V_{2}\right)=\frac{\mu_{2}\left(\left|\nabla^{(2)} V_{2}\right|^{2}\right)}{d_{2}}$ which is a positive constant according to $(H)$, we obtain

$$
(G f)(x, y)=(G f)(x)=-N\left(V_{2}\right) \sum_{i, j=1}^{d_{1}}\left(Q Q^{*}\right)_{i j}\left\{\partial_{x_{i}} \partial_{x_{j}}-\left(\partial_{x_{j}} V_{1}\right)(x) \partial_{x_{i}}\right\} \pi_{1} f(x) .
$$

This enables us to provide the following assertion.

Lemma 3.2. $(I+G)(\mathscr{D})$ is dense in $\mathbb{H}$, so that $(-G, \mathscr{D})$ is essentially m-dissipative (equivalently, essentially self-adjoint) on $\mathbb{H}$.

Proof. First recall that for densely defined, symmetric and dissipative linear operators on a Hilbert space, the property of being essential m-dissipative is equivalent to essential selfadjointness. Consider the operator $\left(T, C_{c}^{\infty}\left(\mathbb{R}^{d_{1}}\right)\right)$ on the Hilbert space $L^{2}\left(\mu_{1}\right)$ defined by

$$
T:=\sum_{i, j=1}^{d_{1}}\left(Q Q^{*}\right)_{i j}\left\{\partial_{x_{i}} \partial_{x_{j}}-\left(\partial_{x_{j}} V_{1}\right)(x) \partial_{x_{i}}\right\} .
$$

Using integration by parts formula we have

$$
\langle T h, g\rangle_{L^{2}\left(\mu_{1}\right)}=-\mu_{1}\left(\left\langle Q Q^{*} \nabla^{(1)} h, \nabla^{(1)} g\right\rangle\right), \quad f \in C_{c}^{\infty}\left(\mathbb{R}^{d_{1}}\right), g \in C^{\infty}\left(\mathbb{R}^{d_{1}}\right) .
$$

By [4, Theorem 7] or [27, Theorem 3.1] our assumptions in $(H)$ imply that $\left(T, C_{c}^{\infty}\left(\mathbb{R}^{d_{1}}\right)\right)$ is essentially self-adjoint (hence, essentially m-dissipative) on $L^{2}\left(\mu_{1}\right)$. Therefore, $L^{2}\left(\mu_{1}\right)=$ $\overline{\mathcal{R}(T)} \oplus \mathcal{N}(T)$. By (1.12) the null space $\mathcal{N}(T)$ consists of the constant functions only. Hence $\left(T, C_{c}^{\infty}\left(\mathbb{R}^{d_{1}}\right)\right)$ restricted to $\mathbb{H}_{1}=\left\{g \in L^{2}\left(\mu_{1}\right): \mu_{1}(g)=0\right\}$ is also essentially self-adjoint. Thus, $(I+G)(\mathscr{D})$ is dense in $\mathbb{H}$, because $\mathbb{H}=\mathbb{H}_{1} \oplus \mathbb{H}_{2}$ and $G$ acts trivial on $\mathbb{H}_{2}$. 
Now we continue to prove the second inequality in (2.15). Let $f \in \mathscr{D}$ and $g=(I+G) f$. As in (3.5), by the definitions of $A$ and $\pi_{1}$ we have

$$
\begin{aligned}
& \left(A^{2} \pi_{1} f\right)(x, y) \\
= & \operatorname{Hess}_{\pi_{1} f}\left(Q \nabla^{(2)} V_{2}\left(y^{\prime}\right), Q \nabla^{(2)} V_{2}(y)\right)(x)-\operatorname{Hess}_{V_{2}}\left(Q^{*} \nabla^{(1)} V_{1}(x), Q^{*} \nabla^{(1)} \pi_{1} f(x)\right)(y) .
\end{aligned}
$$

So,

$$
\begin{aligned}
\left\|A^{2} \pi_{1} f\right\| & \leq\left\|Q \nabla^{(2)} V_{2}\right\|_{L^{4}\left(\mu_{2}\right)}^{2}\left\|\left(\nabla^{(1)}\right)^{2} \pi_{1} f\right\|_{L^{2}\left(\mu_{1}\right)} \\
& +\left\|\left(\nabla^{(2)}\right)^{2} V_{2}\right\|_{L^{2}\left(\mu_{2}\right)}\left\|\left|Q^{*} \nabla^{(1)} V_{1}\right| \cdot\left|Q^{*} \nabla^{(1)} \pi_{1} f\right|\right\|_{L^{2}\left(\mu_{1}\right)} .
\end{aligned}
$$

Due to (3.6) and (3.7) we see that $\pi_{1} f$ solves the elliptic equation

$$
\pi_{1} f-N\left(V_{2}\right) T \pi_{1} f=\pi_{1} g \quad \text { in } L^{2}\left(\mu_{1}\right) .
$$

By applying the elliptic a priori estimates from [9, (2.2) and Lemma 8] (or see [12, Section 5.1] for corresponding proofs including domain issues) to the right hand side of (3.8) we conclude

$$
\left\|(B A)^{*} g\right\|_{L^{2}(\mu)} \leq c\left\|\pi_{1} g\right\|_{L^{2}\left(\mu_{1}\right)} \leq c\|g\|_{L^{2}(\mu)}
$$

for some constant $c \in(0, \infty)$ only depending on $V_{1}$ and $V_{2}$. According to Proposition 2.4 and Lemma 3.2, this implies the second inequality in (2.15). In conclusion, assumption (H3) holds.

Proof of $(H 4)$ : Let $f \in \mathscr{D}(L)$. Since $\mu(f)=0$, we have

$$
\gamma_{1}:=\operatorname{ess}_{\mu} \inf f \leq 0, \quad \gamma_{2}:=\operatorname{ess}_{\mu} \sup f \geq 0 .
$$

Since $\mathscr{D}$ is a core of $(L, \mathscr{D}(L))$, we may take $\left\{g_{n}\right\}_{n \geq 1} \subset \mathscr{D}$ such that $g_{n} \rightarrow f$ and $L g_{n} \rightarrow L f$ in $L^{2}(\mu)$. To control $\left\|g_{n}\right\|_{\text {osc }}$, for any $n \geq 1$ we take $h_{n} \in C^{\infty}(\mathbb{R})$ such that $0 \leq h_{n}^{\prime} \leq 1$ and

$$
h_{n}(r)= \begin{cases}r & \text { for } r \in\left[\gamma_{1}, \gamma_{2}\right], \\ \gamma_{1}-\frac{1}{2 n} & \text { for } r \leq \gamma_{1}-\frac{1}{n}, \\ \gamma_{2}+\frac{1}{2 n}, & \text { for } r \leq \gamma_{2}+\frac{1}{n} .\end{cases}
$$

Then $f_{n}:=h_{n}\left(g_{n}\right) \rightarrow f$ in $L^{2}(\mu)$,

$$
\begin{aligned}
& \limsup _{n \rightarrow \infty}\left\langle-L f_{n}, f_{n}\right\rangle=\limsup _{n \rightarrow \infty} \mu\left(h_{n}^{\prime}\left(g_{n}\right)^{2}\left|\nabla^{(2)} g_{n}\right|^{2}\right) \\
& \leq \limsup _{n \rightarrow \infty} \mu\left(\left|\nabla^{(2)} g_{n}\right|^{2}\right)=\limsup _{n \rightarrow \infty}\left\langle-L g_{n}, g_{n}\right\rangle=\langle-L f, f\rangle,
\end{aligned}
$$

and

$$
\limsup _{n \rightarrow \infty}\left\|f_{n}\right\|_{\text {osc }} \leq \limsup _{n \rightarrow \infty}\left(\gamma_{2}-\gamma_{1}+\frac{1}{n}\right)=\gamma_{2}-\gamma_{1}=\|f\|_{\text {osc }} .
$$

Therefore, we have verified assumption $(H 4)$. 
Proof of Theorem 1.1. It remains to prove (1.8) for $\xi$ in (1.13). Let $\Psi(f)=\|f\|_{\text {osc }}^{2}$ The condition (2.3) is obvious by the definition of $\pi_{1}$ and the $L^{\infty}(\mu)$-contraction of the Markov semigroups $P_{t}$ and $\mathrm{e}^{-t G}$. Since we have verified assumptions $(H 1)-(H 4)$, by Theorem 2.1 it suffices to prove the weak Poincaré inequalities

$$
\begin{gathered}
\left\|\pi_{1} f\right\|^{2} \leq c \alpha_{1}(r)\left\|A \pi_{1} f\right\|^{2}+r \Psi\left(\pi_{1} f\right), \quad r>0, f \in \mathscr{D}\left(A \pi_{1}\right), \\
\left\|\pi_{2} f\right\|^{2} \leq c \alpha_{2}(r)\langle S f, f\rangle+r \Psi(f), \quad r>0, f \in \mathscr{D}
\end{gathered}
$$

for some constant $c \in(0, \infty)$.

Recall that for any $f \in \mathscr{D}$ we have

$$
\left(\pi_{1} f\right)(x, y)=\int_{\mathbb{R}^{d_{2}}} f(x, y) \mu_{2}(\mathrm{~d} y)
$$

By $V_{2}(y)=\Phi\left(|y|^{2}\right)$ we obtain

$$
\begin{aligned}
& \left\|A \pi_{1} f\right\|^{2}=\int_{\mathbb{R}^{d_{1}+d_{2}}}\left\langle Q \nabla^{(2)} V_{2}(y), \nabla^{(1)} \pi_{1} f(x)\right\rangle^{2} \mu(\mathrm{d} x, \mathrm{~d} y) \\
& =\frac{4}{Z\left(V_{2}\right)} \sum_{i, j=1}^{d_{1}} \int_{\mathbb{R}^{d_{1}}}\left(\partial_{x_{i}} \pi_{1} f(x)\right)\left(\partial_{x_{j}} \pi_{1} f(x)\right) \mu_{1}(\mathrm{~d} x) \int_{\mathbb{R}^{d_{2}}} \Phi^{\prime}\left(|y|^{2}\right)^{2}(Q y)_{i}(Q y)_{j} \mathrm{e}^{-\Phi\left(|y|^{2}\right)} \mathrm{d} y \\
& =\frac{4}{Z\left(V_{2}\right)} \sum_{i, j=1}^{d_{1}} \sum_{k=1}^{d_{2}} Q_{i k} Q_{j k} \int_{\mathbb{R}^{d_{1}}}\left(\partial_{x_{i}} \pi_{1} f(x)\right)\left(\partial_{x_{j}} \pi_{1} f(x)\right) \mu_{1}(\mathrm{~d} x) \int_{\mathbb{R}^{d_{2}}} \Phi^{\prime}\left(|y|^{2}\right) y_{k}^{2} \mu_{2}(\mathrm{~d} y) \\
& =\frac{4 \int_{\mathbb{R}^{d}}|y|^{2} \Phi^{\prime}\left(|y|^{2}\right)^{2} \mu_{2}(\mathrm{~d} y)}{Z\left(V_{2}\right) d_{2}} \mu_{1}\left(\left|Q^{*} \nabla^{(1)} \pi_{1} f\right|^{2}\right) .
\end{aligned}
$$

Since $Q Q^{*}$ is invertible, $0<Z\left(V_{2}\right)<\infty$, and

$$
0<4 \int_{\mathbb{R}^{d_{2}}}|y|^{2} \Phi^{\prime}\left(|y|^{2}\right) \mu_{2}(\mathrm{~d} y)=\mu_{2}\left(\left|\nabla^{(2)} V_{2}\right|^{2}\right)<\infty
$$

by $(H)$, this implies

$$
\frac{1}{c} \mu_{1}\left(\left|\nabla^{(1)} \pi_{1} f\right|^{2}\right) \leq\left\|A \pi_{1} f\right\|^{2} \leq c \mu_{1}\left(\left|\nabla^{(1)} \pi_{1} f\right|^{2}\right), \quad f \in \mathscr{D}
$$

for some constant $1<c<\infty$. So, $f \in \mathscr{D}\left(A \pi_{1}\right)$ implies that $\pi_{1} f \in H^{1,2}\left(\mu_{1}\right)$, the completion of $C_{c}^{\infty}\left(\mathbb{R}^{d_{1}}\right)$ under the Sobolev norm $\|g\|_{1,2}:=\sqrt{\mu_{1}\left(g^{2}+\left|\nabla^{(1)} g\right|^{2}\right)}$. Combining this with inequality (1.12) for $i=1$ which naturally extends to $f \in H^{1,2}\left(\mu_{1}\right)$, we prove (3.10).

Next, for the above $f$ and $x \in \mathbb{R}^{d}$, we have $\hat{f}_{x}:=f(x, \cdot)-\pi_{1} f(x) \in C_{c}^{\infty}\left(\mathbb{R}^{d}\right), \mu_{2}\left(\hat{f}_{x}\right)=0$ and $\left\|\hat{f}_{x}\right\|_{\text {osc }} \leq\|f\|_{\text {osc }}$. Then (1.12) for $i=2$ implies

$$
\mu_{2}\left(\left|\hat{f}_{x}\right|^{2}\right) \leq \alpha_{2}(r) \mu_{2}\left(\left|\nabla^{(2)} f(x, \cdot)\right|^{2}\right)+r\|f\|_{\text {osc }}^{2}, \quad r>0 .
$$


Combining this with

$$
\begin{aligned}
& \int_{\mathbb{R}^{d_{1}}} \mu_{2}\left(\left|\hat{f}_{x}\right|^{2}\right) \mu_{1}(\mathrm{~d} x)=\left\|f-\pi_{1} f\right\|^{2}=\left\|\pi_{2} f\right\|^{2}, \\
& \int_{\mathbb{R}^{d_{1}}} \mu_{2}\left(\left|\nabla^{(2)} f(x, \cdot)\right|^{2}\right) \mu_{1}(\mathrm{~d} x)=\int_{\mathbb{R}^{d_{1}+d_{2}}}\left|\nabla^{(2)} f\right|^{2}(x, y) \mu(\mathrm{d} x, \mathrm{~d} y)=-\langle L f, f\rangle,
\end{aligned}
$$

we prove (3.11) for $c=1$.

To prove Example 1.1, we need the following lemma, where the first assertion follows from [20, Theorem 3.1] and Remark(1) after, and the others are taken from [20, Example 1.4] and its proof.

Lemma 3.3. Let $\mu_{V}(\mathrm{~d} x)=\mathrm{e}^{-V(x)} \mathrm{d} x$ be a probability measure on $\mathbb{R}^{d}$. Then the weak Poincaré inequality

$$
\operatorname{Var}_{\mu_{V}}(f) \leq r \alpha_{V}(r) \mu_{V}\left(|\nabla f|^{2}\right)+r\|f\|_{\text {osc }}^{2}, \quad r>0, f \in C_{b}^{1}\left(\mathbb{R}^{d}\right)
$$

holds for some decreasing $\alpha_{V}:(0, \infty) \rightarrow[0, \infty)$. In particular:

(1) If $V(x) \sim k|x|^{\delta}$ or $V(x) \sim k\left(1+|x|^{2}\right)^{\frac{\delta}{2}}$ for some constants $k, \delta>0$, then (3.13) holds with

$$
\alpha_{V}(r)=c\left(\log \left(1+r^{-1}\right)\right)^{\frac{4(1-\delta)^{+}}{\delta}}
$$

for some constant $c>0$.

(2) If $V(x) \sim \frac{d+p}{2} \log \left(1+|x|^{2}\right)$ for some constant $p>0$, then (3.13) holds with

$$
\alpha_{V}(r)=c r^{-\theta(p)}
$$

for some constant $c>0$ and $\theta(p):=\min \left\{\frac{p+d+2}{p}, \frac{4 p+4+2 d}{\left(p^{2}-4-2 d-2 p\right)^{+}}\right\}$.

(3) If $V(x) \sim \frac{d}{2} \log \left(1+|x|^{2}\right)+p \log \log \left(\mathrm{e}+|x|^{2}\right)$ for some constant $p>1$, then (3.13) holds with

$$
\alpha_{V}(r)=c_{1} \mathrm{e}^{c_{2} r^{-\frac{1}{p-1}}}
$$

for some constant $c_{1}, c_{2}>0$.

Proof of Example 1.1. We only consider case (A) and the assertions in the other two cases can be verified in the same way.

By Lemma 3.3, (2.4) holds for

$$
\alpha_{1}(r)=c\left(\log \left(\mathrm{e}+r^{-1}\right)\right)^{\frac{4(1-\delta)^{+}}{\delta}}
$$

for some constant $c>0$. Moreover, for case $\left(A_{1}\right),(2.5)$ holds for

$$
\alpha_{2}(r)=c^{\prime}\left(\log \left(\mathrm{e}+r^{-1}\right)\right)^{\frac{4(1-\varepsilon)^{+}}{\varepsilon}} .
$$


Then for a constant $c_{2}>0$, there exists constants $\kappa_{1}, \kappa_{2}>0$ such that the inequality

$$
c_{2} t \geq \alpha_{1}(r)^{2} \alpha_{2}\left(\frac{r}{\alpha_{1}(r)^{2}}\right) \log \frac{1}{r}
$$

implies

$$
r \leq \kappa_{1} \exp \left(-\kappa_{2} t^{\frac{\delta \varepsilon}{\delta \varepsilon+8 \varepsilon(1-\delta)^{+}+4 \delta(1-\varepsilon)^{+}}}\right) .
$$

Therefore, the desired assertion follows from (1.13).

For case $\left(A_{2}\right)$ we may take

$$
\alpha_{2}(r)=c^{\prime} r^{-\theta(p)}
$$

for some constant $c^{\prime}>0$. Then for a constant $c_{2}>0$, there exists constants $\kappa>0$ such that the inequality (3.15) implies

$$
r \leq \kappa t^{-\frac{1}{\theta(p)}}(\log (\mathrm{e}+t))^{\frac{8(\theta(p)+1)(1-\delta)^{+}+\delta}{\theta(p) \delta}},
$$

so that the desired assertion follows from (1.13).

Finally, for case $\left(A_{3}\right)$ we may take

$$
\alpha_{2}(r)=c^{\prime} \exp \left(c^{\prime \prime} r^{-\frac{1}{p-1}}\right)
$$

for some constants $c^{\prime}, c^{\prime \prime}>0$. Then for a constant $c_{2}>0$, there exists constants $\kappa>0$ such that the inequality (3.15) implies

$$
r \leq \kappa(\log (\mathrm{e}+t))^{-(p-1)} \cdot\left(\log \log \left(\mathrm{e}^{2}+t\right)\right)^{\frac{8(1-\delta)^{+}}{\delta}},
$$

so that the desired assertion follows from (1.13).

\section{References}

[1] F. Baudoin, Bakry-Emery meet Villani, arXiv:1308.4938

[2] A. Bendikov, P. Maheux, Nash-type inequalities for fractional powers of non-negative self-adjoint operators, Trans. Am. Math. Soc. 359(2007), 3085-3097.

[3] L. Beznea, N. Boboc, M. Röckner, Markov processes associated with $L^{p}$-resolvents and applications to stochastic differential equations on Hilbert space, J. Evol. Equat. 6(2006), 745-772.

[4] V. Bogachev, N. V. Krylov, M. Röckner,Elliptic regularity and essential self-adjointness of Dirichlet operators on $\mathbb{R}^{n}$, Ann. Scuola Norm. Sup. Pisa Cl. Sci. (4), 24(1997), 451461.

[5] F. Conrad, M. Grothaus, Construction, ergodicity and rate of convergence of $N$-particle Langevin dynamics with singular potentials, J. Evolu. Equat. 10(2010), 623-662. 
[6] C.-S. Deng, R. Schilling, Y.-H. Song, Sub-geometric rates of convergence for Markov processes under subordination, arXiv:1511.01264.

[7] J. Dolbeault, J. A. Klar, C. Mouhot, C. Schmeiser, Exponential rate of convergence to equilibrium for a model describing fiber Lay-Down processes, Appl. Math. Research eXpress 2013(2013), 165-175.

[8] J. Dolbeault, C. Mouhot, C. Schmeiser, Hypocoercivity for kinetic equations with linear relaxation terms, C. R. Math. Acad. Sci. Paris 347(2009), 511-516.

[9] J. Dolbeault, C. Mouhot, C. Schmeiser, Hypocoercivity for linear kinetic equations conserving mass, Trans. Amer. Math. Soc. 367(2015), 3807-3828.

[10] R. Duan, Hypocoercivity of linear degenerately dssipative kinetic equations, Nonlinearity 24(2011), 2165-2189.

[11] S. Gadat, L. Miclo, Spectral decompositions and $L^{2}$-operator norms of toy hypocoercive semi-groups, Kinetic and related models 6(2013), 317-372.

[12] M. Grothaus, P. Stilgenbauer, Hypocoercivity for kolmogorov backward evolution equations and applications, J. Funct. Anal. 267(2014), 3515-3556.

[13] B. Helffer, F. Nier, Hypoelliptic estimates and spectral theory for Fokker-Planck operators and Witten Laplacians, Lecture Notes in Mathematics, 1862, Springer-Verlag, Berlin, 2005.

[14] J.A. Goldstein, Semigroups of Linear Operators and Applications, Oxford Mathematical Monographs, Oxford University Press, Oxford, 1985.

[15] A. Guillin, F.-Y. Wang, Degenerate Fokker-Planck equations : Bismut formula, gradient estimate and Harnack inequality, J. Diff. Equat. 253(2012), 20-40.

[16] S. Hu, X. Wang, Subexponential decay in kinetic Fokker-Planck equation: weak hypocoercivity, preprint.

[17] A. Nonnenmacher, Essential m-dissipativity of the generator of a generalized stochastic Hamiltonian system and applications, Master thesis, University of Kaiserslautern, 2016.

[18] G. K. Pedersen, Analysis Now, Vol. 118 Grad. Texts Math. Springer-Verlag, New York, 1989.

[19] M. Röckner, $L^{p}$-analysis of finite and infinite-dimensional diffusion operators, Stochastic PDE's and Kolmogorov equations in infinite dimensions (Cetraro, 1998), Lecture Notes in Math. 1715, pp. 65-116, Springer, Berlin, 1999.

[20] M. Röckner, F.-Y. Wang, Weak Poincaré inequalities and $L^{2}$-convergence rates of Markov semigroups, J. Funct. Anal. 185(2001), 564-603. 
[21] R. L. Schilling, J. Wang, Functional inequalities and subordination: stability of Nash and Poincare inequalities, Math. Z. 272(2012), 921-936.

[22] W. Stannat, The theory of generalized Dirichlet forms and its applications in analysis and stochastics, Mem. Amer. Math. Soc. 142(1999), viii+101.

[23] G. Trutnau, Stochastic calculus of generalized Dirichlet forms and applications to stochastic differential equations in infinite dimensions, Osaka J. Math. 37(2000), 315343.

[24] C. Villani, Hypocoercivity, Mem. Amer. Math. Soc. 202(2009), iv+141.

[25] F.-Y. Wang, Hypercontractivity and applications for stochastic Hamiltonian systems, arXiv:1409.1995.

[26] F.-Y. Wang, X. Zhang, Derivative formula and applications for degenerate diffusion semigroups, J. Math. Pures Appl. 99(2013), 726-740.

[27] N. Wielens, The essential self-adjointness of generalized Schrödinger operators, J. Funct. Anal. 61(1985), 98-115. 